\title{
Tele-educação para educação continuada das equipes de saúde da família em saúde mental: a experiência de Pernambuco, Brasil ${ }^{*}$
}

\author{
Magdala de Araújo Novaes ${ }^{1}$ \\ Josiane Lemos Machiavelli² \\ Filipe Cesário Villa Verde ${ }^{3}$ \\ Amadeu Sá de Campos Filho ${ }^{4}$ \\ Tereza Roberta Castro Rodrigues ${ }^{5}$
}

\section{Introdução}

A World Health Organization (WHO) define saúde como um estado de completo desenvolvimento físico, mental e bem-estar social, e não meramente a ausência de doença ou enfermidade (WHO, 1946). Portanto, a saúde mental está intimamente ligada à saúde física e ao comportamento. A saúde está relacionada à promoção do bem-estar, à prevenção dos transtornos mentais, ao tratamento e à reabilitação das pessoas acometidas por transtornos mentais (WHO, 2007).

Estimativas internacionais e do Ministério da Saúde indicam que, no Brasil, aproximadamente cinco milhões de pessoas ( $3 \%$ da população) apresentam transtornos mentais severos e persistentes, necessitando de cuidados contínuos; e nove milhões ( $12 \%$ da população) apresentam transtornos mentais menos graves, necessitando de cuidados eventuais. Em relação aos transtornos decorrentes do uso de álcool, crack e outras drogas, a necessidade de atendimento regular atinge cerca de 6 a $8 \%$ da população, embora existam estimativas ainda mais elevadas. Esses números demonstram que é grande o desafio para os serviços de saúde, especialmente para a atenção primária, porta de entrada do usuário na rede assistencial (Brasil, 2003).

\section{O modelo assistencial em saúde mental brasileiro}

Durante anos, o modelo hospitalocêntrico foi hegemônico na assistência em saúde mental (Facundes et al., 2010; Dimenstein, Galvão, Severo, 2009). Na década de 1960, mais precisamente, as mudanças políticas favoreceram a privatização da assistência em saúde, particularmente da assistência psiquiátrica. Priorizou-se a hospitalização em detrimento da assistência ambulatorial, o que fez aumentar consideravelmente o número de hospitais psiquiátricos no Brasil (Facundes et al., 2010).

Porém, na década de 1940, surgiam propostas na Europa e nos Estados Unidos que buscavam transformar o cuidado psiquiátrico. Tais propostas incluíam as comunidades terapêuticas, psicoterapia de grupo e institucional, psiquiatria de setor, comunitária e preventiva, e a desinstitucionalização (Facundes et al., 2010). A desinstitucionalização não significa apenas desospitalização, mas considerar o

\footnotetext{
Elaborado com base no subprojeto de pesquisa Telessaúde Mental, com coordenação de Magdala de Araújo Novaes, vinculado ao edital: MCT/CNPq No 015/2008 - Institutos Nacionais de Ciência e Tecnologia; pesquisa financiada pelo Conselho Nacional de Desenvolvimento Científico e Tecnológico (CNPq) e pela Fundação de Amparo à Pesquisa do Estado de São Paulo

(Fapesp). O projeto de pesquisa foi aprovado pela Comissão de Ética para Análise de Projetos da Diretoria do Hospital das Clínicas e da

Faculdade de Medicina da Universidade de São Paulo.

1-5 Núcleo de Telessaúde, Universidade Federal de

Pernambuco. Avenida Professor Moraes Rego, $\mathrm{s} / \mathrm{n}$. Hospital das Clínicas, $2^{\circ}$ andar, Cidade Universitária. Recife, $P E$, Brasil. 50.670-420. contato@nutes.ufpe.br
} 
sujeito na sua existência e em relação às suas condições de vida. Reconhece que os portadores de transtornos mentais têm direito a um tratamento efetivo, e não apenas à administração de fármacos ou psicoterapias (Amarante, 1995).

Lentamente, essas experiências passaram a influenciar a assistência psiquiátrica brasileira. $\mathrm{O}$ ano de 1978 costuma ser identificado como o de início efetivo do movimento social pelos direitos dos pacientes psiquiátricos no Brasil. Denominado Reforma Psiquiátrica Brasileira (Brasil, 2005), promoveu ampla mudança no atendimento em saúde mental, garantindo o acesso da população aos serviços e o respeito aos seus direitos e liberdade (Brasil, 2010b). A criação do Sistema Único de Saúde (SUS), por meio da promulgação da Constituição de 1988, possibilitou incluir, nas suas diretrizes, questões associadas à desospitalização e à garantia de cidadania dos doentes mentais (Facundes et al., 2010).

Em 6 de abril de 2001, o Congresso Nacional decretou a Lei 10.216, que dispõe sobre a proteção e os direitos das pessoas portadoras de transtornos mentais, e redireciona o modelo assistencial em saúde mental. O novo modelo prevê que as terapias saiam do escopo medicamentoso exclusivo ou preponderante, de forma que o sujeito ganhe destaque como participante principal do tratamento, juntamente com a família e a sociedade, instaurando um Modelo Psicossocial de Cuidado (Brasil, 2001a).

Em síntese, o paradigma psiquiátrico fundamenta-se no princípio doença-cura, em uma organização de serviços estratificada e hierarquizada, que busca a remissão dos sintomas. O paradigma psicossocial compreende o processo de saúde-doença como algo complexo e que demanda uma abordagem interdisciplinar. Isso faz com que a saúde mental seja situada na saúde coletiva, sendo a integralidade, a intersetorialidade e a territorialidade eixos norteadores das práticas em saúde (Dimenstein, Galvão, Severo, 2009).

Um dos pilares da Reforma Psiquiátrica foi a criação dos serviços substitutivos ao hospital psiquiátrico, tais como: Centros de Atenção Psicossocial (CAPS), leitos psiquiátricos em hospitais gerais, oficinas e residências terapêuticas (Brasil, 2005). O CAPS é considerado o serviço substitutivo mais característico da reforma. Seu objetivo é oferecer acompanhamento clínico e reinserção social dos usuários por meio do trabalho, lazer, exercício dos direitos civis e fortalecimento dos laços familiares e comunitários. Deve ser lugar de referência e tratamento para pessoas acometidas por enfermidades mentais, cuja severidade e/ou persistência justifiquem o cuidado intensivo, comunitário, personalizado e promotor da vida. Apesar de estratégico, o CAPS não deve ser o único tipo de serviço de atenção em saúde mental ofertado no SUS. Isso deve ser feito dentro de uma rede de cuidados, incluindo a atenção primária, mais especificamente a Estratégia de Saúde da Família (Brasil, 2010b; Neves, Lucchese, Munari, 2010; Dimenstein, Galvão, Severo, 2009; Büchele et al., 2006; Brêda et al., 2004; Feneric, Pereira, Zeoula, 2004).

A Saúde da Família pretende transformar o paradigma dominante da queixa/conduta em busca ativa, prevenção e controle das morbidades e promoção da saúde na atenção primária. Além disso, é norteada pelos mesmos princípios que orientam a Estratégia da Atenção Psicossocial (Yasui, Costa-Rosa, 2008) no campo da saúde mental, ou seja, ambas sugerem: a corresponsabilização do cuidado entre profissionais de saúde, usuários e familiares; a proposição de ações intersetoriais, que atuem nos determinantes dos padrões de saúde da população; o desenvolvimento de projetos terapêuticos singulares, que contemplem a diversidade cultural e subjetiva dos usuários; e um modo de trabalho ancorado em práticas de acolhimento e vínculo, superando as perspectivas tecnicistas de trabalho e gestão em saúde (Dimenstein, Galvão, Severo, 2009).

Pesquisas sugerem que é possível resolver $85 \%$ dos problemas de saúde na atenção primária (Brasil, 2001b), o que diminuiria o fluxo intenso de clientes para os setores especializados. Se, em média, um em cada três usuários atendidos nas Unidades de Saúde da Família (USFs) tem transtorno mental, e 50\% dos pacientes atendidos pelos generalistas sofrem somatizações, é importante que as equipes de saúde estejam preparadas para lidar com essas situações (Barban, Oliveira, 2007). Entretanto, a saúde mental sempre foi concebida como especialidade, focada em recursos terapêuticos e na institucionalização. Portanto, sua inclusão na Estratégia de Saúde da Família pode ser caracterizada como uma situaçãocomplexa ou situação-problema a ser superada (Neves, Lucchese, Manari, 2010). A falta de recursos humanos, de conhecimentos sobre a relação entre atenção primária e saúde mental, e/ou de 
capacitações, na área, acabam por prejudicar o desenvolvimento da ação integral pelas equipes de saúde da família, fazendo prevalecer a lógica do encaminhamento. Isso acontece, sobretudo, porque muitos profissionais de saúde não se sentem aptos, confortáveis e seguros para lidar com saúde mental na atenção primária (Dimenstein, Galvão, Severo, 2009; Vecchia, Martins, 2009; Barban, Oliveira, 2007; Nunes, Jucá, Valentim, 2007; Büchele et al., 2006; Brasil, 2005; 2003).

A WHO lembra que, nos países em desenvolvimento, a carência de especialistas e trabalhadores de saúde com os conhecimentos e aptidões necessários para identificação e tratamento dos transtornos mentais constitui uma significativa barreira à prestação de serviços. Além disso, com a integração dos cuidados em saúde mental ao sistema de saúde geral, a tendência é aumentar a procura por generalistas. Logo, é importante que tenham conhecimentos na área de saúde mental (WHO, 2001).

Para superar essas dificuldades, muitas vezes relacionadas à maneira como os profissionais de saúde são formados, é necessário desenvolver neles habilidades para saber fazer, dentre outros, acolhimento, vínculos afetivos e de compromisso com a pessoa e a família em sofrimento psíquico. Os serviços de saúde e as instituições de ensino devem auxiliar nesse processo de transformação (Neves, Lucchese, Manari, 2010) fazendo uso de estratégias que proponham, por exemplo, a capacitação em saúde mental e o apoio aos profissionais que atuam na atenção primária. Nesse contexto, a internet mostra-se como uma possibilidade promissora (Graeff-Martins, 2008; WHO, 2001).

\section{As tecnologias da informação na área da saúde}

As tecnologias da informação, especialmente a internet, por meio das suas redes e comunidades sociais virtuais, são poderosos instrumentos para a comunicação e o acesso às informações sobre saúde (WHO, 2001).

A telessaúde é uma modalidade que vem sendo amplamente utilizada no mundo. Definida como atenção à saúde a distância (WHO, 2010), pode ser útil em diferentes cenários: para dar suporte à decisão clínica, oferecer consultas e diagnóstico a distância - teleassistência; para promover a educação por meio de aulas ou palestras transmitidas em tempo real, por vídeo ou webconferência, ou de forma assíncrona - tele-educação; e, até mesmo, para favorecer a gestão dos serviços de saúde - telegestão (McLaren, 2003). A tele-educação tem sido bastante empregada para proporcionar a educação profissional continuada, especialmente às comunidades localizadas distantes dos grandes centros urbanos (Knowles, 2008; Zollo et al., 1999; Curran, Fleet, Kirby, 2006).

O Brasil dispõe de um Programa Nacional de Telessaúde. Teve início com a publicação da Portaria no 35, de 04 de janeiro de 2007, que instituiu o Projeto Piloto de Telessaúde Aplicado à Atenção Primária e a criação de nove Núcleos de Telessaúde, situados nos estados do Amazonas, Ceará, Pernambuco, Goiás, Minas Gerais, Rio de Janeiro, São Paulo, Santa Catarina e Rio Grande do Sul. Cada Núcleo de Telessaúde conectou-se a cem pontos de telessaúde instalados em Unidades Básicas de Saúde, distribuídos pelo território dos estados. Em 2010, com a Portaria n 402, de 24 de fevereiro, instituiu-se o Programa Telessaúde Brasil em âmbito nacional (Brasil, 2010a), recentemente redefinido e ampliado por meio da Portaria no 2.546, de 27 de outubro de 2011, que o denominou Programa Nacional Telessaúde Brasil Redes (Brasil, 2011).

O Programa Telessaúde Brasil Redes propõe integrar as equipes de saúde da família aos centros universitários de referência para melhorar a qualidade dos serviços prestados na atenção primária, diminuindo os custos da saúde por meio da qualificação profissional, redução da quantidade de deslocamentos desnecessários de pacientes e de profissionais e por meio do aumento de atividades de prevenção das doenças. As ações de apoio à assistência à saúde e de educação permanente das equipes de saúde visam à educação para o trabalho e mudanças de práticas que resultem na qualidade do atendimento na atenção primária (Brasil, 2010a).

O objetivo deste artigo é apresentar as estratégias para a implementação e a avaliação de um serviço de telemedicina, ou telessaúde, os Seminários por Webconferência em Saúde Mental, oferecidos pela Rede de Núcleos de Telessaúde de Pernambuco (RedeNUTES), vinculada ao Programa Telessaúde Brasil Redes, para as equipes de saúde da família. 


\section{A RedeNUTES e sua experiência em Saúde Mental}

Em Pernambuco, o Programa Telessaúde Brasil é desenvolvido, desde 2007, pelo Núcleo de Telessaúde da Universidade Federal de Pernambuco (NUTES-UFPE) por meio da RedeNUTES. A RedeNUTES é fruto do Projeto Telemedicina no Programa Saúde da Família, coordenado pelo Grupo de Tecnologias da Informação em Saúde (Grupo TIS) da Universidade Federal de Pernambuco, com financiamento do Ministério da Saúde em 2001.

A oferta dos serviços de telessaúde (tele-educação e teleassistência) teve início em 2003. A teleeducação foi planejada para capacitar permanentemente as equipes de saúde da família, e a teleassistência, para apoiar a resolução de casos clínicos. Dessa forma, a RedeNUTES implantou, de forma pioneira no Brasil, uma rede de telessaúde voltada para a Estratégia de Saúde da Família, superando inúmeros desafios, tais como: adquirir recursos financeiros para o desenvolvimento de um projeto inovador, conquistar espaço dentro da Universidade para a estruturação de um Núcleo de Telessaúde, e formar recursos humanos que pudessem contribuir na construção de uma rede estadual de telessaúde (Brasil, 2010c).

As conquistas anteriormente citadas permitiram que, a partir de 2005, a RedeNUTES pudesse participar das primeiras discussões que culminariam com a implantação, em 2007, do Projeto Piloto de Telessaúde Aplicado à Atenção Primária. A participação da RedeNUTES nesse projeto possibilitou a manutenção da rede criada em 2001, expandindo-se para mais de cem unidades de saúde da família (Brasil, 2010a).

Os serviços de tele-educação e teleassistência da RedeNUTES são acessados por meio do portal de serviços na internet. Todos os usuários, equipes de saúde e teleconsultores (profissionais que atendem às demandas, a distância, das equipes de saúde) são previamente cadastrados, têm seus acessos monitorados e avaliados pelo NUTES-UFPE. Na teleassistência, são ofertadas teleconsultorias, que possibilitam o esclarecimento de dúvidas relacionadas aos casos clínicos ou ao processo de trabalho na Estratégia de Saúde da Família. Na tele-educação, tem-se o programa de Seminários por Webconferência e o Ambiente Virtual de Aprendizagem da RedeNUTES (AVA RedeNUTES). O serviço de tele-educação visa promover a educação continuada em serviço para as equipes de saúde.

Os seminários consistem em palestras apresentadas de forma síncrona por palestrantes oriundos da UFPE e de outras instituições de ensino, além de profissionais da rede assistencial com expertise no tema que será abordado. As temáticas apresentadas nos seminários são planejadas em função de diferentes critérios: i) avaliação das áreas prioritárias estabelecidas pelo Ministério da Saúde e do perfil epidemiológico de Pernambuco; ii) análise das demandas sugeridas pelas equipes de saúde e pelos gestores parceiros da RedeNUTES; iii) análise das respostas ao formulário de linha de base aplicado durante a implantação do programa nos municípios. Feito isso, a agenda de seminários é elaborada e publicada no portal RedeNUTES para que as equipes de saúde planejem a participação.

São utilizadas duas metodologias para a apresentação dos seminários: exposição do tema por meio de apresentação eletrônica (formato mais comumente utilizado em palestras e aulas), ou discussão de um tema por meio da roda de conversa.

A roda de conversa é uma metodologia participativa, de caráter informal, que possibilita estimular e aprofundar o diálogo sobre um determinado tema. Semelhante às reuniões de grupo, envolve a presença de um moderador, que apresenta o assunto e provoca a participação das pessoas, favorecendo a reflexão, a discussão e a troca de experiências. Pode ser utilizada em diferentes contextos (escolas, unidades de saúde, associações comunitárias, dentre outros), apresenta grande flexibilidade para adaptar os temas centrais às demandas e ao grau de conhecimento dos participantes (Afonso, Abade, 2008), permite coletar informações dos participantes, esclarecer ideias e posicionamentos (Silva, Bernardes, 2007).

Independente do formato utilizado para apresentação dos seminários, sempre existe um tempo para que os participantes possam esclarecer as dúvidas relacionadas ao tema abordado. Cada sessão dura, em média, uma hora. O programa utilizado para transmissão dos seminários é o Adobe ${ }^{\circledR}$ Connect $^{\mathrm{TM}}$, um software proprietário adquirido pelo NUTES-UFPE. Todos os seminários são gravados, editados e armazenados. Posteriormente, são disponibilizados para consulta pela internet por meio do AVA RedeNUTES. 
O ambiente virtual também dispõe das apresentações eletrônicas utilizadas durante os seminários e de materiais complementares (textos, imagens e vídeos) indicados pelos teleconsultores.

Até 2009, a RedeNUTES transmitia quatro seminários por semana nas seguintes áreas temáticas: Saúde da Criança e do Adolescente, Temas Gerais em Saúde Coletiva, Enfermagem e Saúde Mental. Optou-se por trabalhar a Saúde Mental na RedeNUTES porque as equipes de saúde parceiras frequentemente expressam dificuldades para a identificação e o acompanhamento das pessoas com transtornos mentais. Dessa forma, os seminários incentivam a discussão sobre saúde mental; colocam as equipes de saúde em contato com experts da área; apresentam as competências dos profissionais, e discutem as ações possíveis na Estratégia de Saúde da Família.

A definição final dos temas e dos palestrantes que apresentarão os seminários é feita em conjunto com a Gerência de Atenção à Saúde Mental (GASAM) da Secretaria Estadual de Saúde de Pernambuco. O Quadro 1 apresenta a agenda de seminários apresentados ao longo do ano de 2009, totalizando 39 sessões.

\section{A avaliação dos seminários em Saúde Mental}

Ao final de cada sessão, os seminários foram avaliados por meio de um questionário disponibilizado no AVA RedeNUTES. As avaliações ocorreram no período de janeiro a dezembro de 2009, abrangendo as 39 sessões. O Quadro 2 apresenta o modelo de questionário utilizado.

Dos 1422 profissionais que participaram dos seminários, 27\% responderam à avaliação. Os resultados demonstram que: a metodologia utilizada durante os seminários (slides ou roda de conversa) foi aprovada por $97 \%$ dos participantes; $87 \%$ deles se mostraram satisfeitos com a carga horária das sessões (uma hora); os teleconsultores foram considerados ótimos ou bons por $91 \%$ dos respondentes; $82 \%$ classificaram como bom o grau de compreensão dos temas abordados; durante os seminários, $84 \%$ dos participantes não relataram dúvidas sobre o tema abordado ou tiveram suas dúvidas totalmente esclarecidas; $95 \%$ consideraram que os Seminários em Saúde Mental contribuíram para o desenvolvimento das suas atividades profissionais.

Dentre os que responderam sobre os problemas encontrados durante os seminários, o maior inconveniente citado foi a qualidade da conectividade (66\%), que influencia diretamente na qualidade do áudio e do vídeo.

\section{Discussão e conclusões}

As estratégias educativas e terapêuticas na forma de supervisões externas são consideradas eficazes para apoiar o profissional de saúde e potencializar suas ações por meio da reflexão sobre sua atividade cotidiana (Vecchia, Martins, 2009). Além disso, embora não sejam suficientes para transformar as práticas de saúde, podem contribuir para uma progressiva politização e socialização do saber (Silva, Trad, 2005).

Acredita-se que os Seminários em Saúde Mental são uma importante alternativa para favorecer a articulação entre a saúde mental e a atenção primária, oferecendo às equipes de saúde da família, por meio do contato com teleconsultores experientes na área, retaguarda assistencial e suporte técnicopedagógico. E ainda conseguem integrar diversos setores institucionais e intersetoriais (universidades, serviços de saúde, gestão).

Também deve ser comentada a satisfatória adesão dos profissionais de saúde aos seminários que usaram como estratégia a roda de conversa. Essa técnica foi incorporada aos Seminários em Saúde Mental devido à resistência que as equipes de saúde tinham em aceitar a saúde mental como parte das suas atividades profissionais na Estratégia de Saúde da Família e, também, em participar dos serviços de telessaúde que abordavam a temática. Portanto, imaginou-se que oportunizar um espaço para discutir, de forma mais espontânea, saberes e práticas, e para os participantes se conhecerem melhor, poderia facilitar a abordagem. 
Quadro 1. Assuntos apresentados nos Seminários em Saúde Mental da RedeNUTES, janeiro a dezembro de 2009

\begin{tabular}{|c|c|}
\hline Data & Tema \\
\hline 20.01 .09 & Transtornos de humor e de ansiedade \\
\hline 27.01 .09 & Transtornos psicóticos e suicídio \\
\hline 03.02 .09 & Sofrimento psíquico do bebê e saúde mental \\
\hline 10.02 .09 & $\begin{array}{l}\text { Roda de conversa sobre saúde mental na atenção primária } \\
\text { Apresentação do teleconsultor e orientações sobre a atividade proposta }\end{array}$ \\
\hline 17.02 .09 & O CAPS e o trabalho na comunidade \\
\hline 03.03 .09 & Acolhendo o sofrimento psíquico na infância: ações, intervenções e desafios \\
\hline 17.03 .09 & O que é redução de danos? \\
\hline 24.03 .09 & Violência contra a mulher \\
\hline 31.03 .09 & A saúde mental dos profissionais de saúde \\
\hline 07.04 .09 & Hiperatividade em crianças \\
\hline 14.04 .09 & Saúde mental do idoso \\
\hline 28.04 .09 & $\begin{array}{l}\text { Roda de conversa sobre saúde mental na atenção primária } \\
\text { O importante papel do Agente Comunitário de Saúde }\end{array}$ \\
\hline 05.05 .09 & Deficiência de aprendizagem \\
\hline 12.05 .09 & Acolhimento à família do portador de sofrimento psíquico \\
\hline 19.05 .09 & Problemas com as famílias que não assumem seus papéis no tratamento de álcool e outras drogas \\
\hline 02.06 .09 & Atenção à saúde mental infantil: antecedentes históricos e cenário atual \\
\hline 09.06 .09 & CAPSad e Casa do Meio do Caminho \\
\hline 16.06 .09 & Redução de danos na atenção básica \\
\hline 30.06 .09 & $\begin{array}{l}\text { Roda de conversa sobre saúde mental na atenção primária } \\
\text { A promoção da saúde mental }\end{array}$ \\
\hline 07.07.09 & Autismo infantil - Parte I \\
\hline 14.07 .09 & Terapia comunitária \\
\hline 21.07 .09 & Dicas de redução de danos no consumo de drogas lícitas e ilícitas \\
\hline 28.07 .09 & $\begin{array}{l}\text { Roda de conversa sobre saúde mental na atenção primária } \\
\text { A reforma psiquiátrica, o papel do CAPS e a Estratégia de Saúde da Família }\end{array}$ \\
\hline 04.08 .09 & Autismo infantil - Parte II \\
\hline 11.08 .09 & Demências \\
\hline 25.08 .09 & $\begin{array}{l}\text { Roda de conversa sobre saúde mental na atenção primária } \\
\text { Prevenção dos transtornos mentais }\end{array}$ \\
\hline 01.09 .09 & $\begin{array}{l}\text { Roda de conversa sobre saúde mental na atenção primária } \\
\text { O doente mental e sua doença }\end{array}$ \\
\hline 08.09 .09 & Violência doméstica \\
\hline 15.09 .09 & Adolescência e o uso de drogas \\
\hline 22.09 .09 & Como abrir um CAPS \\
\hline 29.09 .09 & $\begin{array}{l}\text { Roda de conversa sobre saúde mental na atenção primária } \\
\text { Esquizofrenia e o papel da equipe de saúde da família }\end{array}$ \\
\hline 06.10 .09 & Prematuramente mãe de bebê prematuro \\
\hline 13.10 .09 & Residências terapêuticas \\
\hline 27.10 .09 & $\begin{array}{l}\text { Roda de conversa sobre saúde mental na atenção primária } \\
\text { Avaliação multiaxial }\end{array}$ \\
\hline 10.11 .09 & Diagnóstico Multiaxial \\
\hline 17.11.09 & Caso clínico de cocomorbidade no consumo de álcool e outras drogas \\
\hline 24.11 .09 & $\begin{array}{l}\text { Roda de conversa sobre saúde mental na atenção primária } \\
\text { Avaliação das rodas de conversa realizadas }\end{array}$ \\
\hline 01.12 .09 & Transtorno do pânico \\
\hline
\end{tabular}

Período: Janeiro a Dezembro de 2009 
Quadro 2. Modelo de questionário utilizado para avaliação dos Seminários, RedeNUTES, 2009

\begin{tabular}{|c|c|c|}
\hline \multicolumn{2}{|r|}{ Município/Formação } & \\
\hline 1 & Aspectos relacionados ao conteúdo/Palestrante & \\
\hline 2 & $\begin{array}{l}\text { Como você classifica a metodologia utilizada durante a } \\
\text { apresentação? }\end{array}$ & $\begin{array}{l}\text { Ótima } \\
\text { Regular } \\
\text { Ruim } \\
\text { Péssima }\end{array}$ \\
\hline 3 & Em relação ao tempo de duração da apresentação, você achou: & $\begin{array}{l}\text { Longo } \\
\text { Curto }\end{array}$ \\
\hline 4 & $\begin{array}{l}\text { Com relação ao palestrante, como você classifica sua } \\
\text { apresentação? }\end{array}$ & $\begin{array}{l}\text { Ótima } \\
\text { Regular } \\
\text { Ruim } \\
\text { Péssima }\end{array}$ \\
\hline 5 & $\begin{array}{l}\text { Como você classifica o seu grau de compreensão do tema } \\
\text { apresentado? }\end{array}$ & $\begin{array}{l}\text { Bom } \\
\text { Razoável } \\
\text { Insuficiente }\end{array}$ \\
\hline 6 & Suas dúvidas foram esclarecidas durante o debate? & $\begin{array}{l}\text { Não tive dúvidas } \\
\text { Tive dúvidas e foram esclarecidas totalmente } \\
\text { Tive dúvidas e foram esclarecidas } \\
\text { parcialmente } \\
\text { Tive dúvidas, mas não foram esclarecidas }\end{array}$ \\
\hline 7 & $\begin{array}{l}\text { Você acredita que a apresentação de hoje vai proporcionar alguma } \\
\text { contribuição para suas atividades profissionais? }\end{array}$ & $\begin{array}{l}\text { Sim } \\
\text { Não } \\
\text { Não sei }\end{array}$ \\
\hline 8 & $\begin{array}{l}\text { De maneira geral, como você classifica esse seminário por } \\
\text { Webconferência? }\end{array}$ & $\begin{array}{l}\text { Ótimo } \\
\text { Regular } \\
\text { Ruim } \\
\text { Péssimo }\end{array}$ \\
\hline 9 & Você indicaria a participação nos seminários a outros profissionais? & $\begin{array}{l}\text { Sim } \\
\text { Não }\end{array}$ \\
\hline & Aspectos Técnicos & \\
\hline 10 & $\begin{array}{l}\text { Qual dos itens à direita dificultaram sua participação nos Seminários } \\
\text { por Webconferência em Saúde Mental? }\end{array}$ & $\begin{array}{l}\text { Áudio } \\
\text { Imagem } \\
\text { Dificuldade no uso do Software (Adobe }{ }^{\circledR} \\
\text { Connect }^{\mathrm{T}} \text { ) } \\
\text { Organização dos Seminários } \\
\text { Infraestrutura } \\
\text { Nenhum }\end{array}$ \\
\hline & Sugestões em Geral & \\
\hline 11 & $\begin{array}{l}\text { Você tem sugestões de temas para os próximos seminários ou } \\
\text { outras sugestões que possam melhorar nosso serviço? }\end{array}$ & [Questão aberta] \\
\hline
\end{tabular}


Nas primeiras sessões, os participantes se demonstraram surpresos ao perceberem que não seria utilizada uma apresentação eletrônica (slides), mas, com o tempo, a surpresa foi dando espaço para maior envolvimento e diálogo. Acredita-se que três fatores foram decisivos para o sucesso da abordagem: definição das temáticas que seriam discutidas a partir das necessidades das equipes de saúde; o teleconsultor-moderador era especialista em psiquiatria, com vasta experiência em atenção primária, saúde da família e atividades em grupo; e, por fim, todas as sessões ocorreram com o mesmo teleconsultor-moderador, o que favoreceu a criação de um vínculo entre ele e as equipes de saúde, que se sentiam confortáveis em expor suas dúvidas e dificuldades.

No entanto, a implementação da tele-educação ainda enfrenta obstáculos em Pernambuco. Um deles é a conectividade. No estado, $80 \%$ dos pontos de telessaúde têm velocidade de conexão igual ou inferior a $256 \mathrm{Kbps}$. O ideal para sessões por webconferência é velocidade superior a $512 \mathrm{Kbps}$. Neste estudo, mais da metade dos profissionais participantes tiveram algum tipo de problema com a internet. Importante ressaltar que a baixa qualidade de conexão não afetou, na maioria das vezes, a parte visual da apresentação dos seminários quando o formato usado era apresentação eletrônica, ou seja, imagens estáticas. Entretanto, nos seminários que usaram roda de conversa ou quando tentou-se transmitir filmes relacionados ao tema em questão, por exemplo, a baixa qualidade da conexão dificultou ou inviabilizou a tentativa.

Outro desafio é a inserção de novas tecnologias no dia a dia dos profissionais de saúde, especialmente dos médicos. Observou-se um baixo percentual de médicos (representaram $5 \%$ dos participantes) nos Seminários em Saúde Mental, entretanto, eles compuseram nosso principal públicoalvo ao longo das sessões. Saliente-se que é na atenção primária que ocorre um contato mais próximo entre os profissionais de saúde e os usuários, pois é a porta de entrada do SUS, logo, deve oferecer oportunidade para o atendimento eficaz. Capacitações contínuas podem contribuir para a melhoria da sua resolubilidade. Considerando-se que, para muitos, essa é a primeira experiência em capacitação mediada pelo computador, acredita-se que é necessário um tempo maior para que entendam a importância do serviço.

Por fim, a produção de conteúdos educativos que realmente façam a diferença para os envolvidos no processo e a avaliação do seu impacto são outros grandes desafios (Geissbuhler, Bagayoko, Ly, 2007). Fatores sociais, econômicos e geográficos podem influenciar a satisfação com os serviços de teleeducação, por isso, a avaliação desses programas é essencial. Poucos trabalhos, no entanto, avaliaram e publicaram experiências dessa natureza, sobretudo em países em desenvolvimento (Kiviat, 2007; Pradeep, 2006), daí a importância deste artigo. Faz-se necessário dar continuidade aos estudos que possibilitem analisar os diferentes aspectos técnicos, socioeconômicos, culturais e políticos que influenciam a introdução da telessaúde como uma prática regular no dia a dia dos serviços, gestores e profissionais de saúde; bem como o impacto da telessaúde no perfil epidemiológico e nos encaminhamentos de pacientes para as redes de referência.

\section{Colaboradores}

Magdala de Araújo Novaes e Josiane Lemos Machiavelli participaram da concepção e desenho do artigo, análise e interpretação dos dados, redação do artigo, revisão crítica do conteúdo intelectual e aprovação final da versão a ser publicada; Amadeu Sá de Campos, Filipe Villa Verde e Tereza Roberta Rodrigues participaram da concepção do artigo, análise e interpretação dos dados, redação do artigo e aprovação final da versão a ser publicada. 


\section{Agradecimentos}

Aos colaboradores do Instituto Nacional de Psiquiatria do Desenvolvimento para Infância e Adolescência (INPD) e da RedeNUTES, vinculada ao Programa Telessaúde Brasil Redes; à Gerência de Atenção à Saúde Mental da Secretaria Estadual de Saúde de Pernambuco; aos teleconsultores que apresentaram os Seminários por Webconferência na área de Saúde Mental.

\section{Referências}

AFONSO, L.; ABADE, F.L. Para reinventar as rodas. Belo Horizonte: Rede de Cidadania Mateus Afonso Medeiros (RECIMAM, 2008). Disponível em: <www.ufsj.edu.br/ portal.../PARA_REINVENTAR_AS_RODAS.pdf>. Acesso em: 14 dez. 2011.

AMARANTE, P. Novos sujeitos, novos direitos: o debate em torno da reforma psiquiátrica. Cad. Saude Publica., v.11, n.3, p.491-4, 1995.

BARBAN, E.; OLIVEIRA, A.A. O modelo de assistência da equipe matricial de saúde mental no programa saúde da família do município de São José do Rio Preto: capacitação e educação permanente aos profissionais de saúde na atenção básica. Arq. Cienc. Saude, v.14, n.1, p.52-63, 2007.

BRASIL. Ministério da Saúde. Portaria № 2.546, de 27 de outubro de 2011. Redefine e amplia o Programa Telessaúde Brasil, que passa a ser denominado Programa Nacional Telessaúde Brasil Redes (Telessaúde Brasil Redes). Brasília, 2011.

. Ministério da Saúde. Secretaria de Gestão do Trabalho e da Educação na Saúde. Programa Telessaúde Brasil Redes. Brasília: Ministério da Saúde, 2010a. Disponível em: <http://www.telessaudebrasil.org.br>. Acesso em: 10 dez. 2010.

. Ministério da Saúde. Saúde Mental. Brasília: Ministério da Saúde, 2010b. Disponível em: <http://portal.saude.gov.br/portal/saude/area.cfm?id_area=925>. Acesso em: 10 dez. 2010.

. Universidade Federal de Pernambuco. Núcleo de Telessaúde. Rede de Núcleos de Telessaúde de Pernambuco. Recife: Universidade Federal de Pernambuco, 2010c. Disponível em: <http://www.redenutes.ufpe.br>. Acesso em: 10 dez. 2010.

. Ministério da Saúde. Secretaria de Atenção à Saúde. Departamento de Ações Programáticas e Estratégicas. Coordenação Geral de Saúde Mental. Reforma psiquiátrica e política de saúde mental no Brasil. Documento apresentado à Conferência Regional de Reforma dos Serviços de Saúde Mental: 15 anos depois de Caracas. Brasília: Ministério da Saúde, 2005.

Ministério da Saúde. Departamento de Atenção Básica. Saúde mental e atenção básica: o vínculo e o diálogo necessários. Brasília: Ministério da Saúde, 2003.

Presidência da República. Casa Civil. Subchefia para Assuntos Jurídicos. Lei $\mathbf{n}^{\circ}$ $\overline{10.216}$, de 06 de abril de 2001. Dispõe sobre a proteção e os direitos das pessoas portadoras de transtornos mentais e redireciona o modelo assistencial em saúde mental. Brasília, 2001a. Disponível em: <http://www.planalto.gov.br/ccivil_03/Leis/ LEIS_2001/L10216.htm>. Acesso em: 10 jan. 2011.

. Ministério da Saúde. Guia Prático do Programa Saúde da Família. Brasília: Ministério da saúde, 2001b.

BRÊDA, M.Z. et al. Duas estratégias e desafios comuns: a reabilitação psicossocial e a saúde da família. Rev. Latino-Am. Enferm., v.13, n.3, p.450-2, 2004.

BÜCHELE, F. et al. A interface da saúde mental na atenção básica. Cogitare Enferm., v.11, n.3, p.226-33, 2006. 
CURRAN, V.R.; FLEET, L.; KIRBY, F. Factors influencing rural health care professionals' access to continuing professional education. Aust. J. Rural Health, v.14, n.2, p.51-5, 2006.

DIMENSTEIN, M.; GALVÃO, V.M.; SEVERO, A.K.S. O apoio matricial na perspectiva de coordenadoras de equipes de saúde da família. Pesqui. Prat. Psicossoc., v.4, n.1, p.37-48, 2009.

FACUNDES, V.L.D. et al. Atenção à saúde mental em Pernambuco: perspectiva histórica e atual. Neurobiology, v.73, n.1, p.183-96, 2010.

FRENERIC, S.R.G.; PEREIRA, W.A.B.; ZEOULA, F.M. Saúde mental e Programa Saúde da Família: percepções do agente comunitário de saúde. Psicol. Hosp., v.2, n.2. Disponível em: <http://pepsic.bvsalud.org/scielo.php?script=sci_arttext\&pid=S167774092004000200011\&lng=pt\&nrm=iso >. Acesso em: 14 dez. 2011.

GEISSBUHLER, A.; BAGAYOKO, C.O.; LY, O. The RAFT network: 5 years of distance continuing medical education and tele-consultations over the Internet in Frenchspeaking Africa. Int. J. Med. Inf., v.76, n.5-6, p.351-6, 2007.

GRAEFF-MARTINS, A.S. et al. Diffusion of efficacious interventions for children and adolescents with mental health problems. JCPP, v.49, n.3, p.335-52, 2008.

KIVIAT, A.D. et al. HIV Online Provider Education (HOPE): the internet as a tool for training in HIV medicine. J. Infect. Dis., v.1, supl.3, p.512-5, 2007.

KNOWLES, L. et al. Disseminating health disparities education through tele-learning. JSCI, v.6, n.4, p.78-82, 2008.

MCLAREN, P. Telemedicine and telecare: what can it offer mental health services? Adv. Psychiatry Treat., n.9, p.54-61, 2003.

NEVES, H.G.; LUCCHESE, R.; MUNARI, D.B. Saúde mental na atenção primária: necessária contribuição de competências. Rev. Bras. Enferm., v.63, n.4, p.666-70, 2010.

NUNES, M.; JUCÁ, V.J.; VALENTIM, C.P.B. Ações de saúde mental no Programa Saúde da Família: confluências e dissonâncias das práticas com os princípios das reformas psiquiátrica e sanitária. Cad. Saude Publica, v.23, n.10, p.2375-84, 2007.

PRADEEP, P.V. et al. Telementoring in endocrine surgery: preliminary Indian experience. Telemed. J. E. Health, v.12, n.1, p.73-7, 2006.

SILVA, P.B.G.; BERNARDES, N.M.G. Rodas de conversa: excelência acadêmica é a diversidade. Educ., v.61, n.1, p.53-92, 2007. Disponível em: < revistaseletronicas.pucrs. br/ojs/index.php/faced/article/view/.../376>. Acesso em: 14 dez. 2011.

SILVA, I.Z.Q.J.; TRAD, L.A.B. O trabalho em equipe no PSF: investigando a articulação técnica e a interação entre os profissionais. Interface - Comunic., Saude, Educ., v.9, n.16, p.25-38, 2005.

VECCHIA, M.D.; MARTINS, S.T.F. Concepções dos cuidados em saúde mental por uma equipe de saúde da família, em perspectiva histórico-cultural. Cienc. Saude Colet., v.14, n.1, p.183-93, 2009.

WORLD HEALTH ORGANIZATION. eHealth. Genebra: WHO, 2010. Disponível em: <http://www.who.int/topics/ehealth/en>. Acesso em: 10 dez. 2010.

What is mental health? Genebra: WHO, 2007. Disponível em: <http://www.who.int/topics/mental_health/en>. Acesso em: 10 dez. 2010.

Relatório mundial da saúde. Saúde mental: nova concepção, nova esperança. Genebra: WHO, 2001. Disponível em: <www.who.int/entity/whr/2001/en/whr01_ djmessage_po.pdf>. Acesso em: $10 \mathrm{dez} .2010$. 
WORLD HEALTH ORGANIZATION. Constitution of the World Health Organization. Am. J. Public Health Nations Health, v.36, n.11, p.1315-23, 1946.

YASUI, S.; COSTA-ROSA, A. A estratégia de atenção psicossocial: desafio na prática dos novos dispositivos de saúde mental. Saude Debate, v.32, n.78-80, p.27-37, 2008.

ZOLLO, S.A. et al. Tele-education in a telemedicine environment implications for rural health care and academic medical centers. J. Med. Syst., v.23, n.2, p.107-22, 1999.

Estudos demonstram que são escassas as ações relacionadas à saúde mental na Estratégia da Saúde da Família, requerendo sensibilização e capacitação dos profissionais. A Telessaúde assume um papel importante, pois favorece a criação de serviços colaborativos entre profissionais de saúde e especialistas trabalhando remotamente. Este artigo apresenta as estratégias para a implementação e a avaliação dos Seminários por Webconferência em Saúde Mental, oferecidos pela RedeNUTES para as equipes de saúde da família de Pernambuco, Brasil. Os seminários síncronos têm frequência semanal e são apresentados por teleconsultores da rede assistencial ou de ensino. As sessões são gravadas e disponibilizadas para consulta na Biblioteca Virtual. Em 2009, ocorreram 39 seminários. A avaliação geral dos seminários, o grau de compreensão dos temas abordados, o material didático utilizado e o tempo de duração tiveram bons resultados. Conclui-se que esse serviço oportuniza um bom espaço para educação continuada em saúde mental.

Palavras-chave: Educação a distância. Saúde mental. Telessaúde. Saúde da família. Avaliação. 


\section{Tele-education for continuing education in mental health for family healthcare teams: an experience in Pernambuco, Brazil}

Studies have shown that actions relating to mental healthcare are rare within the Family Health Strategy. There is a need to raise professionals' awareness and training. Telehealth has taken on an important role because it favors creation of collaborative services between healthcare professionals and specialists working remotely. This paper presents strategies for implementing and evaluating the mental health web conference seminars offered by RedeNUTES for family healthcare teams in Pernambuco, Brazil. The synchronous seminars take place weekly and are presented by teleconsultants from the healthcare or educational network. The sessions are recorded and made available for consultation at the Virtual Library. In 2009, 39 seminars took place. Good results were achieved regarding the overall rating of the seminars, degree of understanding of topics presented, teaching material used and duration of the sessions. It was concluded that this service provided a good space for continuing education on mental health.

Keywords: Distance education. Mental health. Telehealth. Family health. Evaluation.

Tele-educación para la educación continua de los equipos de salud de la familia en salud mental: la experiencia de Pernambuco, Brasil

Hay pocas acciones relacionadas con la salud mental en la Estrategia de Salud de la Familia. Es necesario que los profesionales reciban formación para trabajar con salud mental. La "Telesaúde" tiene un papel importante y favorece la creación de servicios de colaboración entre los profesionales de la salud y especialistas que trabajan de forma remota. Se presentan las estrategias para aplicación y evaluación de los Seminarios por Webconferencia sobre Salud Mental, ofrecidos por la Red Nutes para los equipos de salud de la familia de Pernambuco, Brasil. Los seminarios se realizan en tiempo real. Las sesiones son grabadas y disponibles para inspección en la Biblioteca Virtual. En 2009 ocurrieron se produjo 39 seminarios. La calificación general de los seminarios, el grado de comprensión de los temas, el material didáctico utilizado y el tiempo tuvieron buenos resultados. Se concluye que este servicio ofrece una buena oportunidad para la educación continua en salud mental.

Palabras clave: Educación a distancia. Salud mental. "Telessaúde". Salud de la familia. Evaluación. 